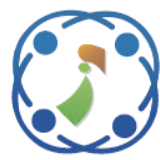

International Journal of

Intelligent Engineering \& Systems

INASS

http://www.inass.org/

\title{
Optimal Placement of Mobile Sink in Wireless Sensor Network Using Adjacency- Based Cell Score Factor
}

\author{
Aravind AR ${ }^{1 *} \quad$ Rekha Chakravarthi ${ }^{1}$ \\ ${ }^{1}$ Sathyabama Institute of Science and Technology, India \\ * Corresponding author's Email: ar123aravind@gmail.com
}

\begin{abstract}
Wireless sensor network (WSN) employs the mobile sink to collect the information from the sensor nodes that are deployed in the network environment in such a way to reduce the energy hole issues. However, the placement of mobile sinks optimally in the network without degrading network lifetime result in a great challenge. Thus, the adjacency-based cell score factor is adopted in this research in order to place the mobile sink optimally in WSN. Here, the network is transformed into the cell using the Voronoi transformation. For each uniform-sized cell, the cluster head $(\mathrm{CH})$ is selected using Sparse-Fuzzy C-means clustering. The optimal placement of the mobile sink is carried out using the adjacency-based cell score, which effectively places the mobile sink using the constrained factors, such as energy, Euclidean distance, and fairness. The proposed adjacency-based cell score obtained better performance with a minimal distance of $22.4615 \mathrm{~m}$, maximal energy, fairness, network lifetime, and throughput of 97.2182, 92.6064, 145, and 95.8852, respectively, while considering 400 nodes.
\end{abstract}

Keywords: Cluster head (CH), Wireless sensor network (WSN), Mobile sink, Path placement, Sparse-FCM.

\section{Introduction}

WSN consist of a large number of mobile or static sensors in a multihop and self-organizing manner. In the recent development of research, the WSN is commonly used in various fields, like smart homes, environmental monitoring, military, building monitoring, and medical health [1]. In water area detection of WSN, the terminal node forwards the information to the sink node through multihop [2] [1]. However, the multihop data transmission may cause the energy hole. As the nodes situated around the sinkhole takes many tasks to forward the data, the nodes may exhaust or consume a lot of energy, and this will damage the network connectivity in WSN [3, 4]. Due to the collision that caused by frequent data transmission and communication between nodes, the multihop transmission produces communication overhead. To solve the above issues, the WSN uses mobile nodes for data transmission. Here, the mobile terminals carry the sink nodes for data collection, which reduces the uneven energy consumption and energy hole, and increases the lifespan of the entire network. Thus, it helps to increase the reliability and flexibility of data transmission [1, 5]. However, the sink mobility is offered as a great solution for sink isolation issue. Mobile sink scatters the energy drainage to the nodes and increases the lifetime of the network by achieving energy consumption [6-8].

To find the optimal number of the sink in WSN is an off-line problem, as it is mainly constrained with the deployment cost. However, determining the placement of the mobile sink optimally results in a more challenging task in WSN. Accordingly, the initial deployment of nodes in WSN can be either done in a planned or structured manner or in a semirandom way. In most cases, the placement of mobile sinks optimally is not known as priori; hence the heuristic is required to facilitate the position of the sink at a new location [9-11]. In [12], the maximumminimum energy consumption approach is developed to find the optimal path between the sink nodes and the network neutron nodes. By introducing the mobile data collector, an algorithm 
based on the cache mechanism is developed in [1] to reduce the $\mathrm{CH}$ overflow and to maximize the network lifetime. However, a mobile data collection mechanism is developed in [13] based on the DSDV routing protocol. Here, the reliability of data communication increases, and the lifespan of the network are extended [1]. In the traditional data collection approach, the nodes are fixed in a certain location in order to collect the data before it being transmitted to the sink node through routing protocol [14-16].

In this research, the optimal placement of the mobile sink is achieved using the adjacency-based cell score. Here, the placement of the mobile sink is carried out by involving three different stages, namely cell network transformation, $\mathrm{CH}$ selection, and mobile sink placement. Initially, the nodes in the WSN are gathered together and the nodes in the network are transformed to the cell using the Voronoi partition. The $\mathrm{CH}$ is selected for each cell in order to make the data transmission easy and reliable. However, the selection of $\mathrm{CH}$ is made using sparse FCM. Finally, the placement of the mobile sink is optimally carried out using the adjacencybased cell score.

The major contribution of this research is discussed below:

- The optimal placement of the mobile sink is achieved using the adjacency-based cell score in such a way that the lifespan of the network is extended and the energy consumed by the nodes is reduced based on the factors, like the location of the sink, fairness, energy, and Euclidean distance.

The paper is organized as follows: section 2 discusses the review of various existing mobile sink placement methods, and section 3 elaborates the proposed adjacency-based cell score factor for placing the mobile sink in WSN. Section 4 discusses the result and discussion and finally, section 5 concludes the paper.

\section{Motivation}

In this section, some of the existing methods for mobile sink placement are discussed, which motivate the researchers to develop a new mechanism for optimally placing the mobile sink.

\subsection{Literature survey}

Various existing techniques are surveyed in this section. Chao, F et al. [1] developed a mobile data collection mechanism in the sensor network in order to collect the monitoring data more flexibly and efficiently. It used the clustering algorithm for dynamically grouping the terminal nodes, which helped to minimize energy consumption. However, the angular bisector approach was used to find the path of unmanned aerial vehicles (UAVs). Moreover, the correctness and the effectiveness of this method were proved through the simulated results. It failed to verify the reliability of the path. Vijayashree, R. and Suresh Ghana Dhas, C [16] introduced an artificial bee colony $(\mathrm{ABC})$ algorithm for finding the data collection path in the mobile sink. This algorithm greatly increased the lifetime of the network. It effectively reduced energy consumption and increased data collection. It increased the throughput and efficiently collects the data in order to save energy. However, it failed to use the data fusion approach with the sensor node.

Amar Kaswan et al. [19] developed a model named as, multi-objective particle swarm optimization (MOPSO), for the mobile sink placement. This method was used to select the best global and local solutions in the search space, which offered better results in statistical significance. The optimization of multiple mobile sinks was the major drawback of this method. Praveen Kumar et al. [20] proposed a method for the path determination of mobile sink based on the Ant Colony Optimization (ACO). This method offered better results in energy consumption and network lifetime. Anyhow, handling of multiple mobile sinks with non-uniform data constraints were difficult.

Saunhita Sapre, and S. Mini [21] developed an optimization algorithm, named Differential Moth Flame Optimization (DMFO), in which traversal strategy was used for the mobile sink placement. This method offered better results in energy consumption and network lifetime but failed to calculate the data latency and throughput. Elie T. Fute et al. [22] proposed an instantaneous clustering algorithm (ICP) for the determination of the target points from the mobile sink. The main aim of this method was to reduce the clustering time and increasing the network's lifetime. The communication overhead was the major disadvantage of this method.

\subsection{Challenges}

Some of the challenges associated with the placement of mobile sink are as follows:

Energy hole is a major challenging issue in WSN as the sensor nodes situated around the sink node attain higher traffic load and it results in energy depletion [16]. Due to the limited self-energy of sensor nodes, the network poses a communication overhead problem [16]. As the nodes in WSN have limited energy capability and the nodes are located 
in harsh ambiance, hence it is difficult to recharge or replace the node's battery [18]. In the case of the static sink, the sink mobility poses a great challenge, as tracking the mobile sink in the area of sensing is hard to achieve.

\section{Proposed optimal placement of the mobile sink}

To place the mobile sink optimally in WSN without degrading the network lifetime and energy consumption poses a major challenging task. Hence, an adjacency-based cell score is formed in this research to perform the mobile sink placement optimally. Initially, the nodes are simulated in the WSN and they are transformed into the cell. The cell network transformation is carried out using the Voronoi partition. After performing the cell network transformation, the $\mathrm{CH}$ is selected for each cell in the network using sparse FCM. Finally, the placement of the mobile sink is optimally achieved using the adjacency-based cell score. Fig. 1 portrays the schematic diagram of the optimal placement of the mobile sink.

\subsection{Cell Network Transformation}

Initially, the nodes in the WSN are grouped together in order to transform the nodes of the cell network. The network is transformed into various cells using Voronoi partition, which determines the optimal partitioning of cells in the WSN environment. The collection of different cell regions are specified as $A_{m}$ such that $(1 \leq m \leq P)$, which indicates that there exist $P$ regions are generated based on the $M_{1, \ldots} M_{n}$. However, the network that is transformed using the Voronoi partition is termed as a cell network. The transformed cell network is then passed to the $\mathrm{CH}$ selection phase in order to select the $\mathrm{CH}$ from each partitioned region. Table 1 depicts the symbols and the definition of those symbols used in this paper.

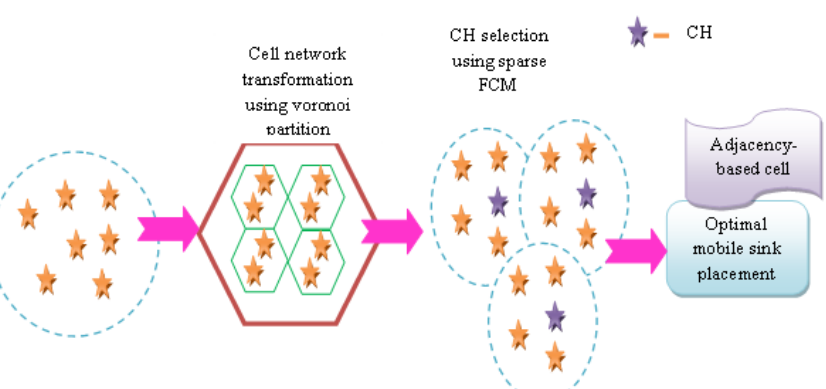

Figure. 1 Schematic diagram of optimal placement of the mobile sink
Table 1. Symbol Table

\begin{tabular}{|c|l|}
\hline Notation & \multicolumn{1}{|c|}{ Definition } \\
\hline$u$ & Number of data points \\
\hline$v$ & Number of attributes \\
\hline$p$ & Weight of the attributes \\
\hline$L$ & Cluster center \\
\hline$c a r d(q)$ & Cardinality of set $q$ \\
\hline$\beta$ & Dissimilarity measure \\
\hline$p_{r}$ & Objective function \\
\hline$G_{l}$ & Class \\
\hline$T$ & Number of cells \\
\hline$F_{o}$ & Placement of sink at $o^{\text {th }}$ cell \\
\hline$F_{S}$ & Placement of sink at $s^{t h}$ cell \\
\hline$H()$. & Euclidean distance \\
\hline$L o c_{0}$ & Location of sink at $o^{\text {th }}$ cell \\
\hline$L o c_{S}$ & Location of sink at $s^{\text {th cell }}$ \\
\hline$T^{e}$ & Maximum number of nodes \\
& sharing resources equally \\
\hline$E_{S}$ & Fairness \\
\hline$d$ & Tuning parameter \\
\hline
\end{tabular}

\subsection{CH Selection Using Sparse-FCM}

Voronoi transformation then, it is required to select the $\mathrm{CH}$ in each region in order to make the placement of the mobile sink more optimal. Here, the $\mathrm{CH}$ selection is carried out using sparse FCM, which is derived with the inclusion of the FCM algorithm with sparse regularization. The SparseFCM solves the issues associated with the data clustering.

Algorithm. 1 Pseudo code of Sparse-FCM for $\mathrm{CH}$ selection

\begin{tabular}{|r|l|}
\hline S1. No & \multicolumn{1}{|c|}{ Sparse-FCM for CH selection } \\
\hline $\mathbf{1}$ & $\begin{array}{l}\text { Input: } x \text { cluster, and data matrix as } X_{b}= \\
W_{y z}^{b}=\beta^{u \times v}\end{array}$ \\
\hline $\mathbf{2}$ & $\begin{array}{l}\text { Output: cluster centroid } \\
\left\{B_{1}, B_{2}, \ldots . B_{i}, \ldots . B_{x}\right\} \text { and } p^{a}\end{array}=$ \\
\hline $\mathbf{3}$ & Begin \\
\hline $\mathbf{4}$ & Initialize $p=p_{1}^{a}=p_{2}^{a}=\cdots=p_{v}^{a}=\frac{1}{\sqrt{v}}$ \\
\hline $\mathbf{5}$ & Compute $R$ \\
\hline $\mathbf{6}$ & Specify $S$ \\
\hline $\mathbf{7}$ & Fix $S$ and $R$ and calculate $G_{l}$ \\
\hline $\mathbf{8}$ & Compute $p^{*}$ \\
\hline $\mathbf{9}$ & Terminate \\
\hline
\end{tabular}


However, the cluster centroids ( $B)$ generated using Sparse-FCM is represented in Eq. (1).

$$
B=\left\{B_{1}, B_{2}, \ldots . B_{i}, \ldots . B_{x}\right\}
$$

Let us consider the data matrix as $X_{b}=W_{y z}^{b}=$ $\beta^{u \times v}$ such that $(1 \leq y \leq u)$ and $(1 \leq z \leq v)$. The dimension of $b^{\text {th }}$ data matrix is specified as $[u \times$ $v$ ] and the rows and columns of $X_{b}$ is represented as $W_{y}^{b} \epsilon \beta^{v}$ and $W_{z}^{b} \epsilon \beta^{u}$, respectively. The Sparse-FCM selects the $\mathrm{CH}$ based on the minimum distance between the cluster centroid and individual data points. However, the algorithmic steps involved in the Sparse-FCM are specified in algorithm 1.

The algorithmic steps involved in the SparseFCM to select the $\mathrm{CH}$ from the partitioned cell region are elaborated as follows:

i) Initialization: Let us initialize the weights of the attributes as, $p=p_{1}^{a}=p_{2}^{a}=\cdots=p_{v}^{a}=\frac{1}{\sqrt{v}}$

ii) Update partition matrix: Let us specify the $p$ and $L$ such that $\beta$ is minimized using the following condition shown in Eq. (2).

$$
R_{j w}=\left\{\begin{array}{c}
\frac{1}{g_{j}} ; K_{w}=0, \text { and } g_{j}=\operatorname{card}(q) \\
0 ; K_{w} \neq 0 \text { but } K_{j t}=0 \\
\frac{1}{\sum_{t=1}^{h} \frac{K_{w} \frac{1}{K_{j t}}}{f-1}} ; \text { otherwise }
\end{array}\right.
$$

However, the distance measured in the sparse FCM is mathematically denoted in Eq. (3).

$$
K_{w}=\sum_{j=1}^{h} p_{r}\left(S_{w r}-S_{j r}\right)^{2}
$$

The distance between the data points and the cluster center is computed using the above equation.

iii) Update the cluster center: Let $p$ and $\beta$ be the set and $\varepsilon(B)$ is minimized if

$$
B=\left\{\begin{array}{c}
0 \quad ; \text { if } p_{r}=0 \\
\frac{\sum_{t=1}^{h} R_{j w}-S_{w r}}{\sum_{j=1}^{h} R_{j w}} ; \text { if } p_{r} \neq 0
\end{array}\right.
$$

Where, $j=1, \ldots . x$.

iv) Compute the class: The class value is calculated based on $R$ and $B$. However, the $G_{l}$ is represented as, $\max \sum_{i=1}^{v} p_{l}$. $G_{l}$ such that $\|p\|_{2}^{2} \leq$ $1,\|p\|_{k}^{k} \leq d$ and compute $p^{*}$.

v) Terminate: The above steps are repeated until the solution is obtained or met the stopping criteria.
Therefore, the cluster centroid obtained using the Sparse-FCM is specified in Eq. (5).

$$
\frac{\sum_{l=1}^{v}\left|p_{l}^{*}-p_{l}^{a}\right|}{\sum_{l=1}^{v}\left|p_{l}^{a}\right|}<10^{-4}
$$

\subsection{Optimal Placement of mobile sink node}

Once the $\mathrm{CH}$ is selected using sparse FCM then, the placement of the mobile sink process is performed optimally in WSN. The optimal placement of the mobile sink is achieved using the adjacency based cell score, which effectively places the mobile sink using the constrained factors, such as energy, Euclidean distance, and fairness. By using this, the lifespan of the network is extended and the energy consumed by the nodes is reduced. Also, this method uses the minimum distance, which leads to reduce energy consumption. Let us consider $T$ and TCH's in the network, which is represented in Eq. (6).

$$
F=\left\{F_{1}, F_{2}, \ldots . F_{o}, \ldots . F_{T}\right\} ; 1 \leq o \leq T
$$

However, the placement of sink from $F_{o}$ to $F_{S}$ is based on the adjacency-based cell score and is specified in Eq. (7).

$$
\min A d_{s}=J_{s}+H_{s}+\left(1-E_{s}\right)
$$

Where,

$$
\begin{aligned}
& J_{S}=\frac{1}{t} \sum_{\propto=1}^{t} J_{\text {loss }}^{\text {predict }}(\propto) \\
& H_{S}=H\left(\operatorname{Loc}_{o}, \operatorname{Loc}_{S}\right)
\end{aligned}
$$

Moreover, the $E_{S}$ is represented in Eq. (10).

$$
\boldsymbol{E}_{\boldsymbol{s}}=\frac{T^{e}}{T}
$$

\section{Results and discussion}

The results and discussion obtained by the proposed adjacency-based cell score is discussed in this section with respect to the performance metrics.

\subsection{Experimental setup}

The implementation of the proposed adjacencybased cell score for placing the mobile sink in WSN is carried out in the network simulator-2 (NS-2) using 200, 300, and 400 nodes by varying the simulation rounds. 


\subsection{Evaluation metrics}

The performance improvement of the proposed approach is evaluated using the metrics, such as distance, residual energy, normalized fairness, network lifetime, and normalized throughput, respectively.

\subsection{Comparative methods}

The performance of the proposed model is revealed by comparing the proposed with the existing methods, like Ant Colony Optimizationbased Mobile Sink Path determination (ACOMSPD) [20], Multi-Objective Particle Swarm Optimization (MOPSO) [19], Differential Moth Flame Optimization (DMFO) [21], and Instantaneous clustering algorithm (ICP) [22], respectively.

ACO-MSPD [20]: In this method, the paths of the mobile sinks are determined based on the ACO, in which the near-optimal sets of rendezvous points are calculated. OPSO [19]: In this method, the paths of the mobile sinks are calculated based on the derivation of a proficient multi-objective fitness function and the particle encoding scheme. Also, the MOPSO is used to calculate the global and local best solutions of the particles.

DMFO [21]: In this method, a traversal strategy for the mobile sink is considered for the collection of sensed data. The sink movement is calculated based on the exponential complexity of the Traveling Salesman Problem (TSP).ICP [22]: In this method, the path of the mobile sink is calculated based on the circumference visit method, which is used to minimize the clustering time and increase the network lifetime.

\subsection{Comparative analysis}

The comparative analysis of the proposed adjacency-based cell score is discussed in this section by varying the number of rounds with respect to the simulation rounds.

\section{a) Analysis based on 200 nodes}

Fig. 2 portrays the comparative analysis of the proposed adjacency-based cell score using 200 nodes. Fig. 2 (a) represents the analysis of distance with respect to the number of rounds. The minimum distance is considered as the best performance. At round-500, the distance of the mobile sink is 44.533 for ACO-MSPD, 44.314 for MOPSO, 38.585 for FROA, 41.320 for DMFO, 41.063 for ICP, and 35.243 for the proposed adjacency-based cell score. At round 1000 the mobile sink distance of the proposed method is 70.847 , which is $14.22 \%, 7.25 \%$,
$4.86 \%, 9.33 \%$, and $5.99 \%$, better than the existing methods, such as ACO-MSPD, MOPSO, F-ROA, DMFO, and ICP, respectively.

Fig. 2 (b) represents the analysis of residual energy with respect to the number of rounds. At round-500, the energy consumed by the mobile sink is 86.188 for ACO-MSPD, 89.200 for MOPSO, 88.6737 for F-ROA, 91.5856 for DMFO, 91.4917 for ICP, and 92.1331 for the proposed adjacencybased cell score. Similarly, for 750 rounds, the proposed method has a maximum residual energy of 76.933. The percentage of improvement with the existing methods, such as ACO-MSPD, MOPSO, DMFO, and ICP, is $2.89 \%, 2.67 \%, 2.97 \%$, and $2.64 \%$, respectively.

Fig. 2 (c) represents the analysis of normalized fairness with respect to the number of rounds. At round-500, the normalized fairness of the existing methods, like ACO-MSPD, MOPSO, F-ROA, DMFO, and ICP is 76.475, 77.703, 78.905, 75.917, and 78.149 , while the proposed adjacency-based cell score achieved the normalized fairness of 86.22 , respectively. Likewise, the maximum normalized fairness attained by the proposed method is 58.029, for 1500 rounds. For the same round the existing methods, such as ACO-MSPD, MOPSO, F-ROA, DMFO, and ICP, is attain the normalized fairness of 53.96, 51.794, 55.116, 49.023, and 51.84, respectively, which is minimum than the proposed method.

Fig. 2 (d) represents the analysis of network lifetime with respect to the number of rounds. The maximum value is considered as the best performance of network lifetime. At round-500, the network lifetime of the existing methods, like ACOMSPD, MOPSO, F-ROA, DMFO, and ICP is 37,38 , 40, 36.886, and 39.37, while the proposed adjacency-based cell score have the network lifetime of 41 , respectively. Similarly, for 750 rounds, the network lifetime attained by the methods, such as ACO-MSPD, MOPSO, F-ROA, DMFO, ICP, and the proposed adjacency-based cell score is, 36, 34, $36,33.072,35.955$, and 37, respectively, in which the proposed method offers maximum network lifetime.

Fig. 2 (e) represents the analysis of normalized throughput with respect to the number of rounds. At round-500, the normalized throughput obtained by the existing methods, like ACO-MSPD, MOPSO, FROA, DMFO, and ICP is 84.056, 85.194, 87.965, 84.977 , and 87.43 , while the proposed adjacencybased cell score obtained better throughput of 88.222 , respectively. Likewise, by considering 2000 rounds, the normalized throughput obtained by the proposed adjacency-based cell score 54.255. For the 


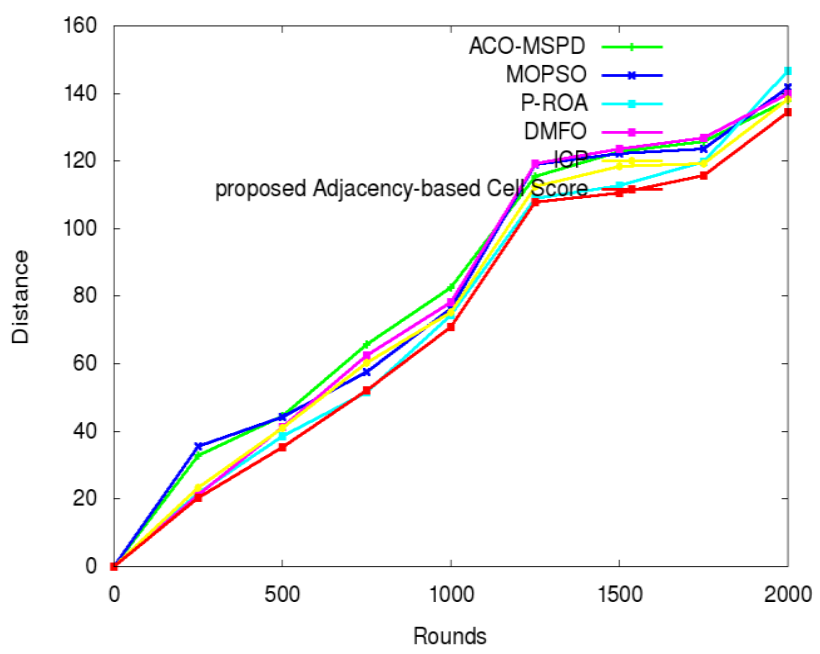

(a)

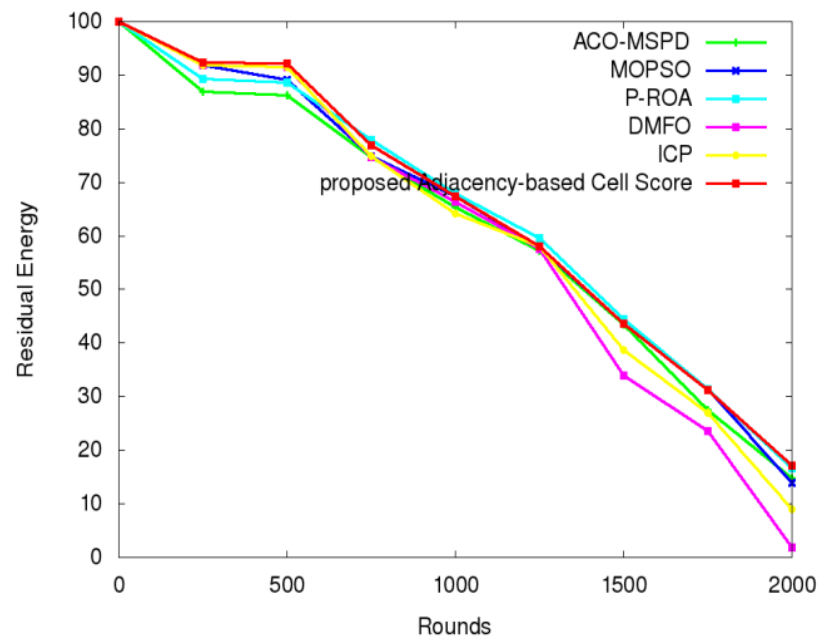

(b)

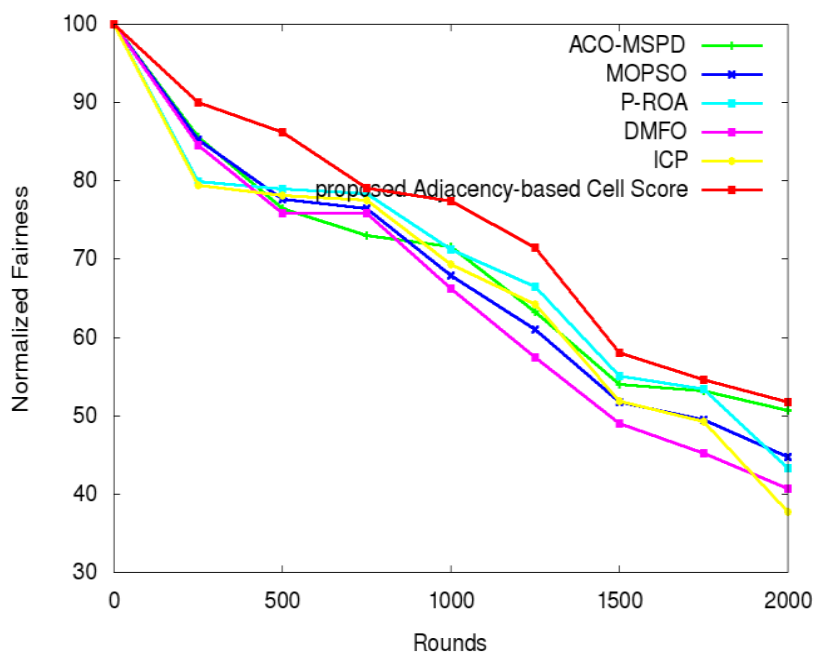

(c)

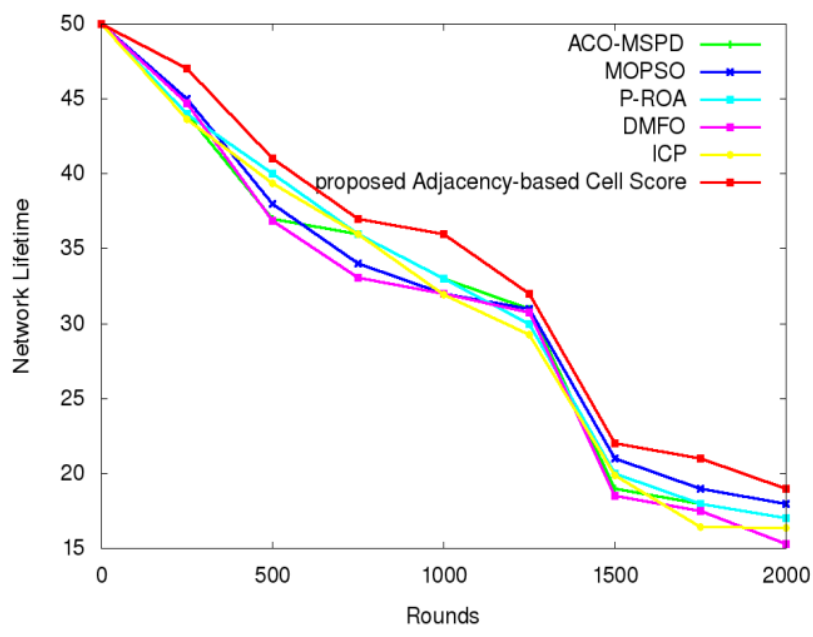

(d)

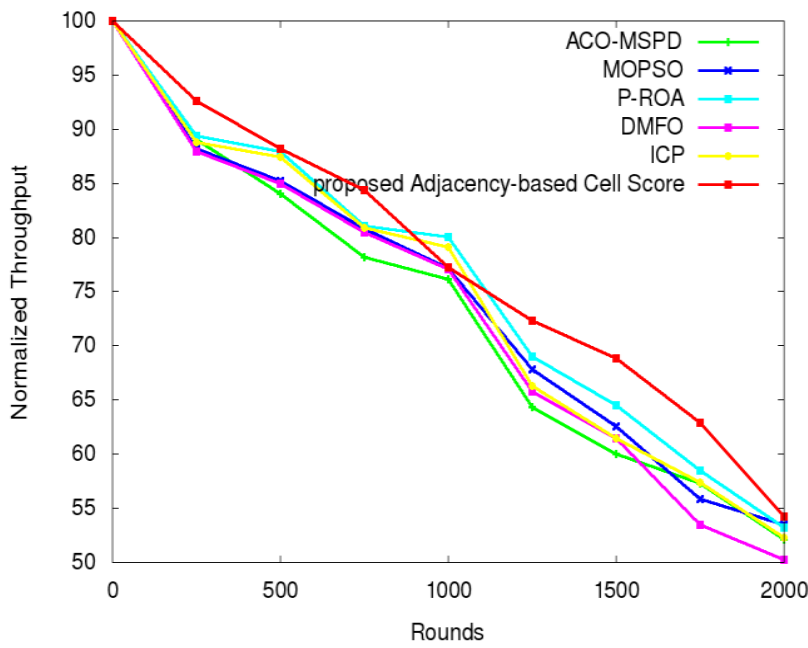

(e)

Figure. 2 Analysis based on 200 nodes: (a) distance (b) residual energy (c) normalized fairness (d) network lifetime (e) normalized throughput

same round the normalized throughput obtained by the existing methods, such as ACO-MSPD, MOPSO, F-ROA, DMFO, and ICP is 52.156, 53.49, 53.259, 50.256 , and 52.285. Here, the maximum value is considered as the best normalized throughput.

\section{b) Analysis based on 300 nodes}

Fig. 3 portrays the comparative analysis of the proposed adjacency-based cell score using 300 nodes. Fig. 3 (a) represents the analysis of distance with respect to the number of rounds. At round-500, the distance of the mobile sink is 42.609 for ACOMSPD, 49.280 for MOPSO, 43.704 for F-ROA, 41.814 for DMFO, 38.997 for ICP, and 38.594 for 


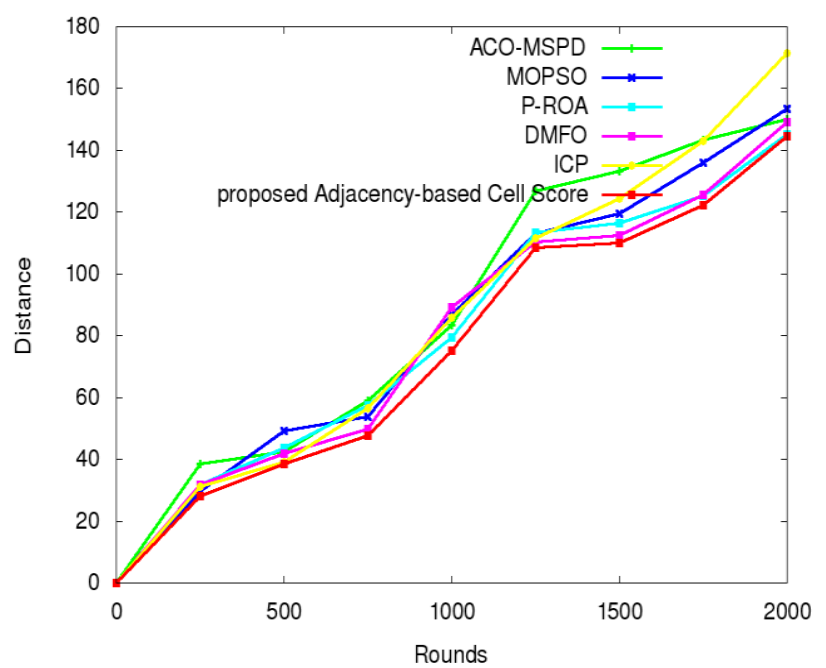

(a)

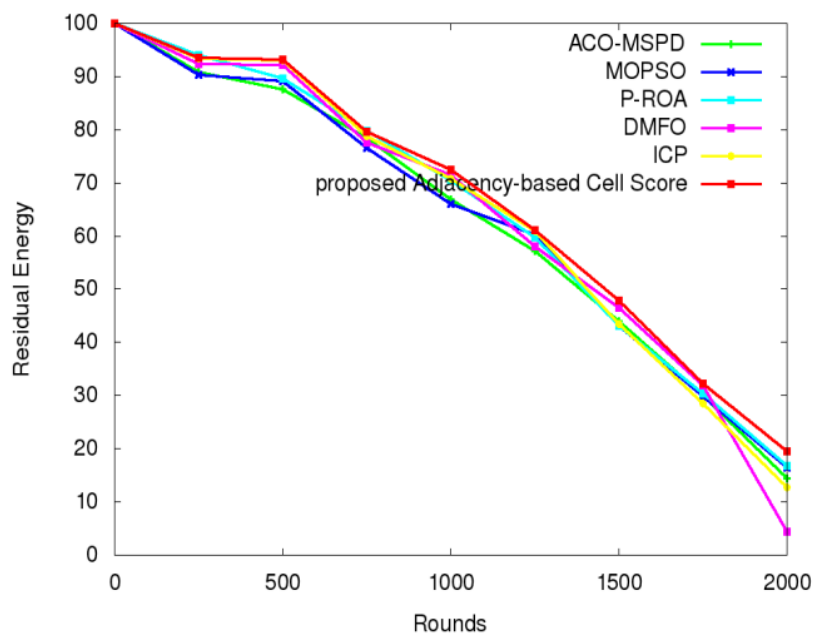

(b)

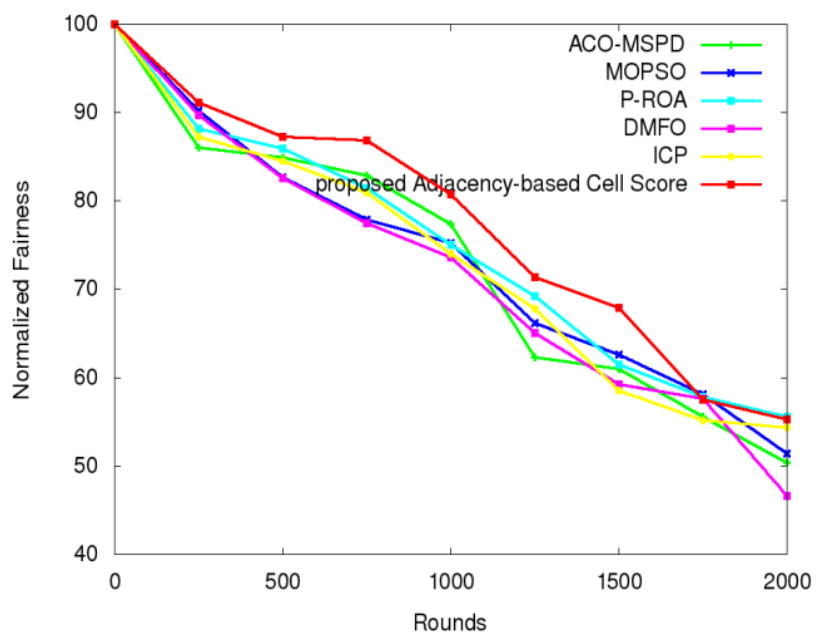

(c)

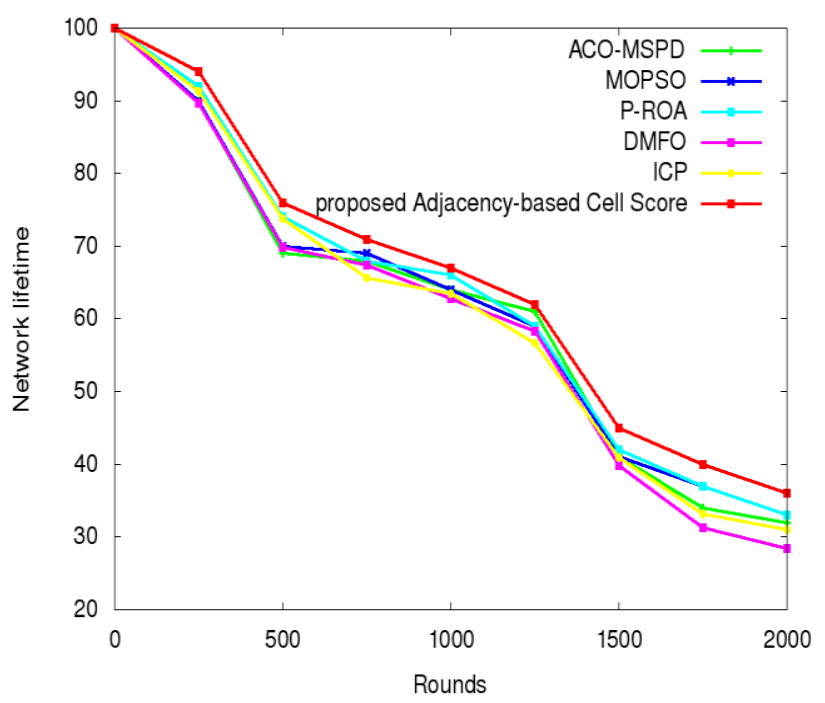

(d)

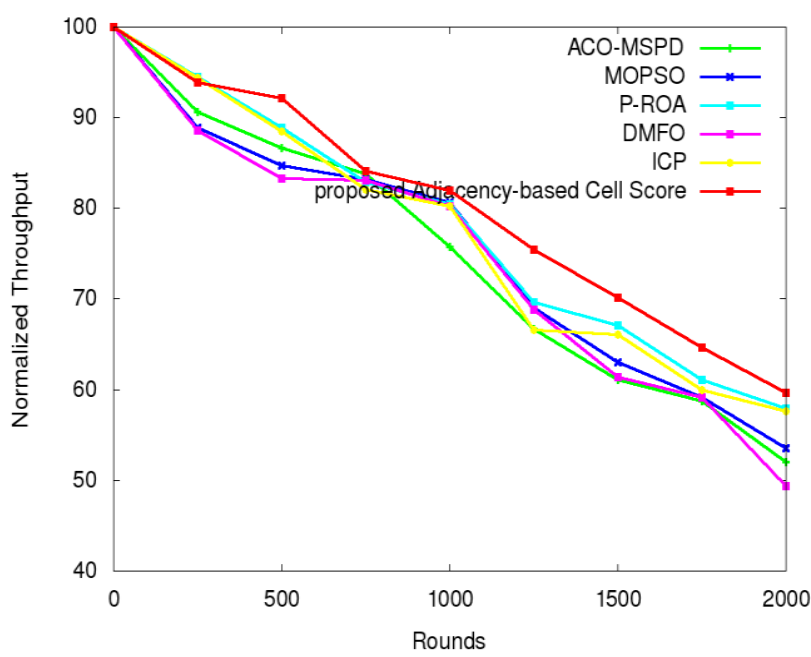

(e)

Figure. 3 Analysis based on 300 nodes: (a) distance (b) residual energy (c) normalized fairness (d) network lifetime (e) normalized throughput

the proposed adjacency-based cell score. At round750 , the distance of the mobile sink is 59.029 for ACO-MSPD, 53.914 for MOPSO, 57.362 for FROA, 49.754 for DMFO, 56.686 for ICP, and 47.786 for the proposed adjacency-based cell score.

Fig. 3 (b) represents the analysis of residual energy with respect to the number of rounds. At round-500, the energy consumed by the mobile sink is 87.673 for ACO-MSPD, 89.1209 for MOPSO, 89.5694 for F-ROA, 92.222 for DMFO, 93.055 for ICP, and 93.278 for the proposed adjacency-based cell score. At round-750, the energy consumed by the mobile sink is 78.409 for ACO-MSPD, 76.566 for MOPSO, 79.825 for F-ROA, 77.626 for DMFO, 78.762 for ICP, and 79.709 for the proposed adjacency-based cell score. 
Fig. 3 (c) represents the analysis of normalized fairness with respect to the number of rounds. At round-500, the normalized fairness of the existing methods, like ACO-MSPD, MOPSO, F-ROA, DMFO, and ICP is 84.966, 82.698, 85.901, 82.604, and 84.492 , while the proposed adjacency-based cell score achieved the normalized fairness of 87.281, respectively. Similarly, at round-750, the normalized fairness of the existing methods, like ACO-MSPD, MOPSO, F-ROA, DMFO, and ICP is $82.93,77.85$, $81.471,77.458$, and 80.938 , while the proposed adjacency-based cell score achieved the normalized fairness of 86.8723 , respectively.

Fig. 3 (d) represents the analysis of network lifetime with respect to the number of rounds. At round-500, the network lifetime of the existing methods, like ACO-MSPD, MOPSO, F-ROA, DMFO, and ICP is 69, 70, 74, 69.83, and 73.73, while the proposed adjacency-based cell score has the network lifetime of 76, respectively. Likewise, the network lifetime of the existing methods, like ACO-MSPD, MOPSO, F-ROA, DMFO, and ICP is $68,69,68,67.382$, and 65.614 , while the proposed adjacency-based cell score has the network lifetime of 71, respectively, for the rounds 750 .

Fig. 3 (e) represents the analysis of normalized throughput with respect to the number of rounds. At round-500, the normalized throughput obtained by the existing methods, like ACO-MSPD, MOPSO, FROA, DMFO, and ICP is 86.662, 84.671, 88.850, 83.299 , and 88.508 , while the proposed adjacencybased cell score obtained better throughput of 92.13, respectively. Similarly, at round-1000, the normalized throughput obtained by the existing methods, like ACO-MSPD, MOPSO, F-ROA, DMFO, and ICP is 75.76, 80.659, 80.443, 80.191, and 80.245 , while the proposed adjacency-based cell score obtained better throughput of 81.9227, respectively.

\section{c) Analysis based on $\mathbf{4 0 0}$ nodes}

Fig. 4 portrays the comparative analysis of the proposed adjacency-based cell score using 400 nodes. Fig. 4 (a) represents the analysis of distance with respect to the number of rounds. At round-500, the distance of the mobile sink is 58.032 for ACOMSPD, 46.101 for MOPSO, 47.15 for F-ROA, 34.783 for DMFO, 36.807 for ICP, and 31.589 for the proposed adjacency-based cell score. Similarly, at round-750, the distance of the mobile sink is 62.515 for ACO-MSPD, 65.573 for MOPSO, 57.668 for F-ROA, 56.84 for DMFO, 52.663 for ICP, and 49.327 for the proposed adjacency-based cell score. Here, the minimum value is considered as the best distance.

Fig. 4 (b) represents the analysis of residual energy with respect to the number of rounds. At round-500, the energy consumed by the mobile sink is 91.550 for ACO-MSPD, 92.4006 for MOPSO, 90.093 for F-ROA, 94.743 for DMFO, 95.258 for ICP, and 95.413 for the proposed adjacency-based cell score. Likewise, at round-750, the energy consumed by the mobile sink is 81.525 for ACOMSPD, 81.713 for MOPSO, 81.427 for F-ROA, 82.347 for DMFO, and 83.595 for ICP, and 84.535 for the proposed adjacency-based cell score.

Fig. 4 (c) represents the analysis of normalized fairness with respect to the number of rounds. At round-500, the normalized fairness of the existing methods, like ACO-MSPD, MOPSO, F-ROA, DMFO, and ICP is $81.165,86.951,90.342,85.993$, and 90.235 , while the proposed adjacency-based cell score achieved the normalized fairness of 91.24, respectively. At round-750, the normalized fairness of the existing methods, like ACO-MSPD, MOPSO, F-ROA, DMFO, and ICP is 80.876, 85.0939, $88.8419,85.017$ and 88.711, while the proposed adjacency-based cell score achieved the normalized fairness of 89.22 respectively.

Fig. 4 (d) represents the analysis of network lifetime with respect to the number of rounds. At round-500, the network lifetime of the existing methods, like ACO-MSPD, MOPSO, F-ROA, DMFO, and ICP is $113,115,112,112.428$, and 110.6, while the proposed adjacency-based cell score has the network lifetime of 114 , respectively. At round-750, the network lifetime of the existing methods, like ACO-MSPD, MOPSO, F-ROA, DMFO, and ICP is 106, 107, 108, 102.826, and 107.135, while the proposed adjacency-based cell score has the network lifetime of 111, respectively, which is maximum than other existing methods.

Fig. 4 (e) represents the analysis of normalized throughput with respect to the number of rounds. At round-500, the normalized throughput obtained by the existing methods, like ACO-MSPD, MOPSO, FROA, DMFO, and ICP is $90.414,92.3718,90.714$, 92.002, and 90.617, while the proposed adjacencybased cell score obtained better throughput of 94.561, respectively. Likewise, at round-1000, the normalized throughput obtained by the existing methods, like ACO-MSPD, MOPSO, F-ROA DMFO, and ICP is $79.261,83.985,83.658,82.733$, and 82.377 , while the proposed adjacency-based cell score obtained better throughput of 84.798 respectively. 


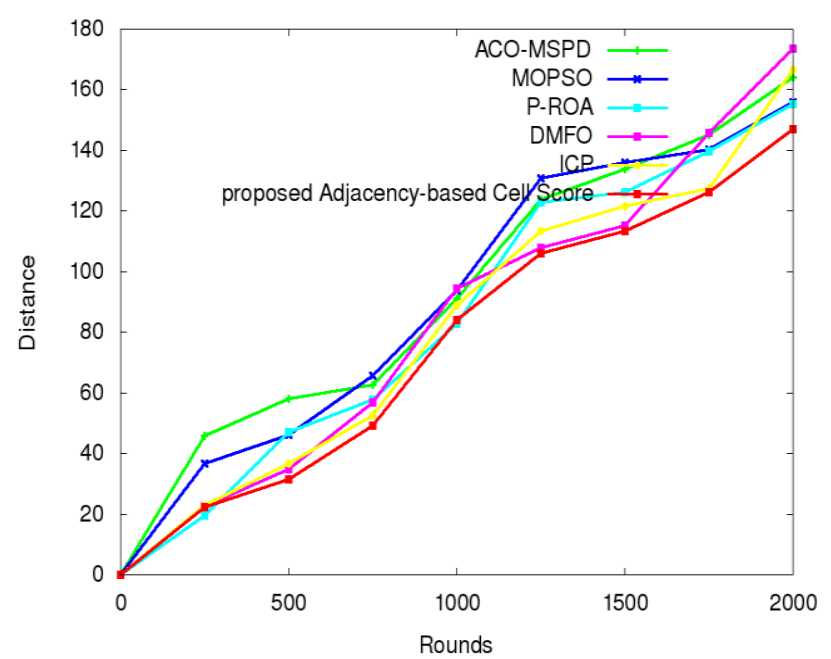

(a)

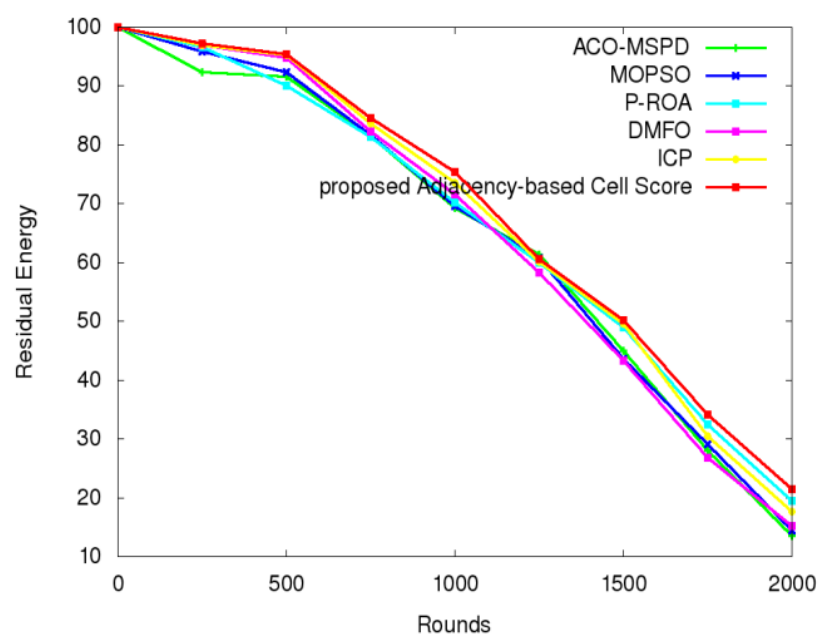

(b)

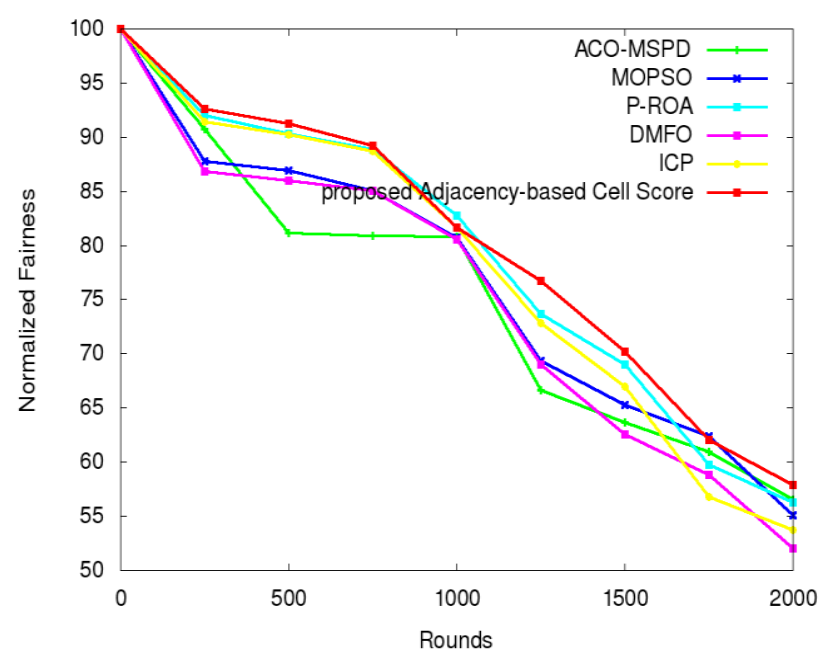

(c)

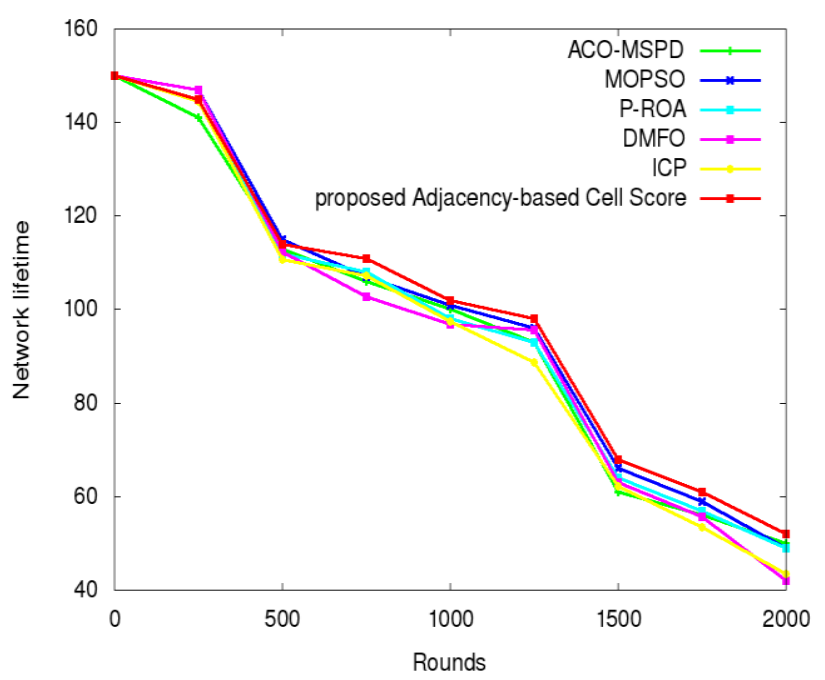

(d)

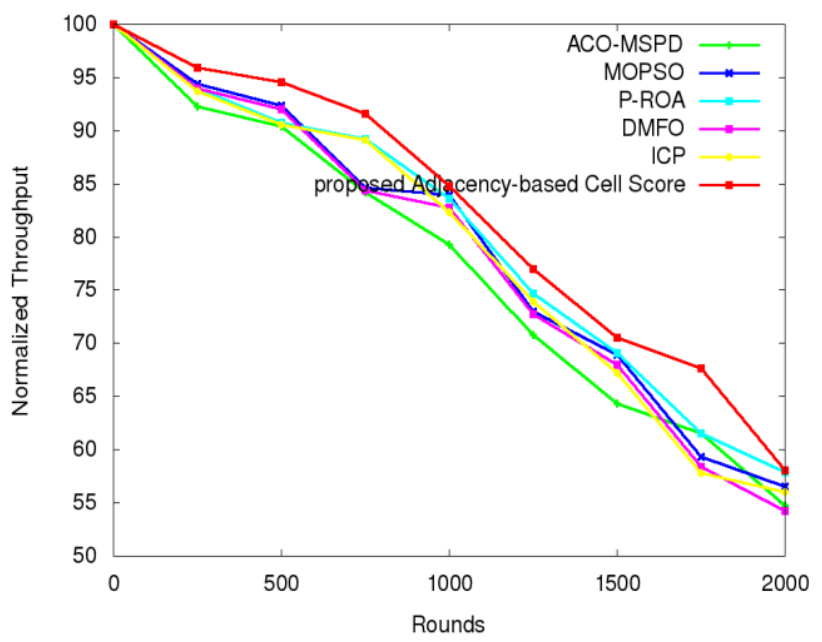

(e)

Figure. 4 Analysis based on 400 nodes: (a) distance (b) residual energy (c) normalized fairness (d) network lifetime (e) normalized throughput

\subsection{Comparative discussion}

The comparative analysis is carried out by considering the 200, 300, and 400 nodes, in which the best performance occurs at 250 rounds. In the proposed method, the minimum distance occurs, when considering the 200 nodes. For 200 nodes, the distance of the proposed method is 20.429 , which is $37.83 \%, 42.65 \%, 5.49 \%, 3.24 \%$, and $13.02 \%$, better than the existing methods, such as ACO-MSPD, MOPSO, F-ROA, DMFO, and ICP, respectively. By considering the metrics, such as residual energy, normalized fairness, network lifetime, and normalized throughput, the maximum that is 400 nodes offers the best performance. The maximum residual energy attained by the proposed method is 97.2182. The percentage of improvement of the proposed method with the existing methods, such as 
ACO-MSPD, MOPSO, F-ROA, DMFO, and ICP, is $4.97 \%, \quad 1.32 \%, 0.62 \%, 0.3 \%$, and $0.22 \%$, respectively. For normalized fairness, in the existing method, F-ROA attained the maximum performance of 92.0208 , but the proposed system is $0.63 \%$, better than the existing F-ROA. Similarly, the existing method MOPSO offered maximum normalized throughput of 94.3683, but the proposed method is $1.58 \%$ better than the existing MOPSO. Thus, the proposed adjacency-based cell score method outperforms than the existing methods, such as ACO-MSPD, MOPSO, F-ROA, DMFO, and ICP, respectively.

In the proposed method, the network is transformed into various cells using Voronoi partition, which determines the optimal partitioning of cells in the WSN environment. Also, the $\mathrm{CH}$ selection is carried out using sparse FCM, which is derived with the inclusion of the FCM algorithm with sparse regularization. The optimal placement of the mobile sink is achieved using the adjacency based cell score, which effectively places the mobile sink using the constrained factors, such as energy, Euclidean distance, and fairness. By using this, the lifespan of the network is extended and the energy consumed by the nodes is reduced. Also, this method uses the minimum distance, which leads to reduce energy consumption. Thus, the proposed method offers better results, than the existing methods.

\section{Conclusion}

In this research, the optimal placement of the mobile sink is dealt with the adjacency-based cell score using the factors, like energy, distance, and fairness. Initially, the nodes are simulated in the WSN environment. The sensor nodes in the network are transformed into the cell with uniform size and shape such that the cell network transformation is carried out using the Voronoi partition. Thereby, the $\mathrm{CH}$ is selected for each uniform-sized cell in the network in order to make the data transmission more reliable and effective. The selection of $\mathrm{CH}$ is carried out using the sparse FCM. After selecting the $\mathrm{CH}$, the optimal placement of the mobile sink is performed using the adjacency-based cell score. However, the placement of the mobile sink is done based on the factors, like energy, Euclidean distance, and fairness. The proposed adjacency-based cell score obtained better performance with a minimal distance of $22.4615 \mathrm{~m}$, maximal energy, fairness, network lifetime, and throughput of 97.2182, 92.6064, 145, and 95.8852, respectively, for considering 400 nodes. Environmental monitoring,
Border protection, and crowd management are some of the applications of the proposed method. In the future, the performance of the proposed system will be further enhanced by using a recent optimization algorithm.

\section{Conflicts of Interest}

The author declares no conflict of interest.

\section{Author Contributions}

Conceptualization, AR Aravind and Rekha Chakravarthi; methodology, AR Aravind and Rekha Chakravarthi; software, AR Aravind and Rekha Chakravarthi; writing-review and editing, AR Aravind and Rekha Chakravarthi.

\section{Acknowledgment}

The authors are grateful to the management of Sathyabama Institute of Science and Technology, Chennai that greatly assists the research and development.

\section{References}

[1] F. Chao, Z. He, A. Pang, H. Zhou, and J. Ge,"Path Optimization of Mobile Sink Node in Wireless Sensor Network Water Monitoring System", Complexity, Hindawi, Vol. 2019, pp. 1-10, 2019.

[2] R. Yue and T. Ying, "A water quality monitoring system based on wireless sensor network \& solar power supply", In: Proc. of IEEE International Conf. On Cyber Technology in Automation, Control, and Intelligent Systems, Kunming, China, pp. 126-129, 2011.

[3] P. P. G. Neogi, "IWD-based Mobile Sink Route Determination for WSN under Non-Uniform Data Constraints", In: Proc. of IEEE International Conf. On Computer Communication and Informatics (ICCCI), Coimbatore, India, pp. 1-6, 2019.

[4] T. S. Chen, W. Q. Du, and J. J. Chen, "EnergyEfficient Data Collection by Mobile Sink in Wireless Sensor Networks", In: Proc. of IEEE Wireless Communications and Networking Conference (WCNC), Marrakech, Morocco, pp. 1-6, 2019.

[5] R. W. Pazzi and A. Boukerche, "Mobile data collector strategy for delay-sensitive applications over wireless sensor networks", Computer Communications, Vol. 31, No. 5, pp.1028-1039, 2008. 
[6] J. Wang, Y. Gao, W. Liu, A. K. Sangaiah, and H. J. Kim, "Energy Efficient Routing Algorithm with Mobile Sink Support for Wireless Sensor Networks", Sensors, Vol. 19, No. 7, p.1494, 2019.

[7] N. A. Shiltagh, M. Z. Abdullah, and A. R. Zarzoor, "Evaluation of routing protocol with multi-mobile sinks in WSNs using QoS and energy consumption parameters", International Journal of Electrical \& Computer Engineering, Vol. 9, No. 4, pp.2880-2892, 2019.

[8] V. Kumar and A. Kumar, "Improving reporting delay and lifetime of a WSN using controlled mobile sinks", Journal of Ambient Intelligence and Humanized Computing, Vol. 10, No. 4, pp.1433-1441, 2019.

[9] H. Ramadan and D. P. Agrawal, "Effective data routing using mobile sinks in disjoint mobile wireless sensor networks", Periodicals of Engineering and Natural Sciences, Vol. 7, No. 1, pp.82-98, 2019.

[10] M. Krishnan, S. Yun, and Y. M. Jung, "Enhanced clustering and ACO-based multiple mobile sinks for efficiency improvement of wireless sensor networks", Computer Networks, Vol. 160, pp.33-40, 2019.

[11] J. Flathagen, Ø. Kure, and P. E. Engelstad, "Constrained-based multiple sink placement for wireless sensor networks", In: Proc. of IEEE Eighth International Conference On Mobile Ad-Hoc and Sensor Systems, Valencia, Spain, pp. 783-788, 2011.

[12] N. Ghosh and I. Banerjee, “An energy-efficient path determination strategy for mobile data collectors in wireless sensor network", Computers \& Electrical Engineering, Vol. 48, pp.417-435, 2015.

[13] Chatzigiannakis, A. Kinalis, and S. Nikoletseas, "Efficient data propagation strategies in wireless sensor networks using a single mobile sink", Computer Communications, Vol. 31, No. 5, pp.896-914, 2008.

[14] N. Gharaei, S. J. Malebary, K. A. Bakar, S. Z. M. Hashim, S. A. Butt, and G. Sahar, "EnergyEfficient Mobile-Sink Sojourn Location Optimization Scheme for Consumer Home Networks", IEEE Access, Vol. 7, pp.112079112086, 2019.

[15] M. Kang, I. Yoon, and D. K. Noh, "Efficient Location Service for a Mobile Sink in SolarPowered Wireless Sensor Networks", Sensors, Vol. 19, No. 2, p.272, 2019.

[16] R. Vijayashree and C. S. Ghana Dhas, "Energy efficient data collection with multiple mobile sink using artificial bee colony algorithm in large-scale WSN", Automatika Journal for Control, Measurement, Electronics, Computing and Communications, Vol. 60, No. 5, pp.555563, 2019.

[17] L. Zhang and C. Wan, "Dynamic Path Planning Design for Mobile Sink with Burst Traffic in a Region of WSN", Wireless Communications and Mobile Computing, Hindawi, Vol. 2019, pp. 1-8, 2019.

[18] V. Chauhan and S. Soni, "Mobile sink-based energy efficient cluster head selection strategy for wireless sensor networks", Journal of Ambient Intelligence and Humanized Computing, pp.1-14, 2019.

[19] D. P. Kumar, A. Tarachand, A. Chandra, and S. Rao, "ACO-based mobile sink path determination for wireless sensor networks under non-uniform data constraints", Applied Soft Computing, Vol. 69, pp. 528-540, 2018.

[20] A. Kaswan, V. Singh, and P. K. Jana, "A multiobjective and PSO based energy efficient path design for mobile sink in wireless sensor networks", Pervasive Mobile Computing, Vol. 46, pp. 122-136, 2018.

[21] S. Sapre and S. Mini, "A differential moth flame optimization algorithm for mobile sink trajectory", Peer-to-Peer Networking and Applications, pp. 1-14, 2020.

[22] T. Elie, Z. Aline, and E. Tonye, "A Reliable and Efficient Path Discovery Method for Mobile Sink based Wireless Sensor Networks", International Journal of Computer Applications, Vol. 176, No. 25, pp.17-22, 2020. 